\title{
DPART - A DIGITAL TWIN FRAMEWORK FOR THE MACHINING DOMAIN
}

\author{
P. Ganser ${ }^{1 *}$, T. Venek ${ }^{1}$, V. Rudel ${ }^{1}$, T. Bergs ${ }^{1,2}$ \\ ${ }^{1}$ Fraunhofer Institute for Production Technology IPT, Dept. High Performance Cutting, Aachen, Germany \\ ${ }^{2}$ Laboratory for Machine Tools and Production Engineering (WZL) of RWTH Aachen University, Aachen, Germany \\ ${ }^{\star}$ Corresponding author; e-mail: philipp.ganser@ipt.fraunhofer.de
}

\begin{abstract}
Todays' heterogeneous manufacturing environments and isolated manufacturing elements hinder the realization of a complete and data consistent digital twin. Against this background, an increased connectivity based on the Industrial Internet of Things (IloT) might be the future key enabler for the digital twin. However, it requires each domain to transfer, rearrange and rethink their individual data solutions in a framework that is IloT-ready. This paper presents an IloT-based implementation of a digital twin framework for machining, enabling the creation of a complete and data consistent digital twin throughout process planning, manufacturing and quality assurance. Different use cases are introduced based on the example of a blade integrated disk for modern turbofan engines.
\end{abstract}

\section{Keywords:}

Digitalization, Digital Twin, Industrial Internet of Things, Machining, Milling, Turning

\section{INTRODUCTION}

Today, digitization is the primary driver for innovations in manufacturing. In the context of digitization the digital twin, a virtual representation of a physical manufacturing element, is the central object of interest. However, within the existing heterogeneous manufacturing environments and their isolated manufacturing elements, creation of a complete and data consistent digital twin is a challenging task. In the near future, an increased connectivity between all relevant manufacturing elements in the sense of the Industrial Internet of Things (IloT) might be the key enabler for the digital twin. In "Digital Factories 2020" PwC states, that the use of connectivity technologies and big data analytics in manufacturing is set to increase dramatically until 2022 and beyond [Geissbauer 2017]. Against this background, each domain is responsible to transfer, rearrange and rethink their individual data solutions in a digital twin framework that is lloT-ready.

As one of the most important domains in manufacturing, machining (especially cutting) benefits from a long-term course of development and an advanced level of digitization. At the same time, it is characterized by the interaction of various software and hardware components for planning and execution of the manufacturing process. Examples are computer-aided manufacturing systems (CAM), computerized numerically controlled machine tools (CNC), or coordinate measurement machines (CMM). However, the data generated within the software and hardware components of machining is so far been used almost exclusively to fulfill the individual task of the single component. As a result, process planning, manufacturing and quality assurance nowadays work on different, only extremely incomplete digital twins. A cross-system solution, which couples process planning, manufacturing and quality assurance, and creates a complete and data-consistent digital twin, is missing. Correlations and causalities, as well as optimization potentials regarding quality, time, costs and sustainability remain unexposed and unused.

Against the introduced problem, this paper presents a domain-specific implementation of a digital twin framework for machining (called dPart®), enabling a complete and data consistent digital twinning throughout process planning, manufacturing and quality assurance. The implementation comprises data collection, processing and analysis functionalities in an IloT infrastructure for a targeted evaluation and optimization of machining processes. The proposed framework enhances the completeness and the benefits of the digital twin, increases the digital resilience in machining and prepares it for a future data economy.

\section{STATE OF THE ART}

The modern idea of the digital twin dates back to 2003, when Grieves introduced his "Conceptual Ideal for PLM" in the context of Product Lifecycle Management (PLM). The idea described the connection of the real and virtual space (and its objects) through data and information exchange along the four phases of creation, production, operation and disposal of a product. Initially, the concept carried the name "Mirrored Spaces Model" or "Information Mirroring Model". 
At a later stage, Grieves and his co-author Vickers introduced the name Digital Twin. [Grieves 2015]

Within the last two decades, the idea of the digital twin has emerged into various sectors apart from PLM, such as construction, healthcare and many more [Glaessgen 2012, Boje 2020, Croatti 2020]. The sheer number of different application sectors and stakeholders has led to a large variety of definitions for the digital twin, along with terms such as the digital shadow [Abele 2016, Negri 2017 , Kritzinger 2018, Bergs 2020a, Bergs 2021]. Today, concerning manufacturing, the general definition of Grieves still resembles the fundamental idea of the digital twin, it being a virtual representation of a physical product.

Within the development towards the "perfect" digital twin in manufacturing, the initial level of digitization within the addressed technology domain plays an important role. As introduced beforehand, machining, with its broad industrial application, advanced state of the art and mature software and hardware components, benefits from an advanced digitization level. Over the last decades, a number of concepts and technologies has paved the way towards today's status of digitization in machining.

As an example, virtual manufacturing systems (VMS) or virtual process systems (VPS) enhance the completeness of the digital twin, especially in the planning phase of production, based on the integration of coupled simulation tools within CAM [Brecher 2011, Altintas 2014, Wiederkehr 2016]. Cyber-physical (production) systems (CPS/CPPS) connect computational entities with the physical world of production, providing data accessing and data processing services available on the internet, thereby building a cyberphysical environment for the digital twin, especially in the manufacturing phase of production [acatech 2011, Monostori 2014]. Today, it is the concept of industry 4.0 , smart manufacturing and big data, along with its technologies such as loT, edge and cloud computing, and artificial intelligence (Al), which further accelerates digitization in manufacturing, thereby significantly enhancing the maturity of the digital twin.

Hänel et al. propose a digital twin for machining processes for the application in aerospace industries [Hänel 2020]. The method is able to create a digital twin with increased information content from planning and process data in machining. The underlying information model structures data acquisition, model-based data processing and data storage via Hierarchical Data Format (HDF) technology, along with visualization functionalities. It is demonstrated based on the example of a lever component from the aerospace sector. Caesar et al. introduce the corresponding information model [Caesar 2020].

Teige et al. address a system for the intelligent, networked and autonomous manufacturing of aerospace components [Teige 2021]. The system connects the process steps of cutting tool manufacturing, autonomous machining and application. It acquires high-frequency machine data from manufacturing devices via an edge solution and forwards them to an Al solution on cloud level. Incorrect process conditions are detected and corrective instructions are returned to the field level. The concept of the digital twin is not directly addresses. However, the system provides connectivity approaches in the sense of 14.0 and loT.

Armendia et al. focus on the generation of a digital twin of the machine tool and the application for machine tool and machining process optimization [Armendia 2019a]. Based on a virtual machine tool model, the system is able simulate machining operations and to reduce machine tool and machining process setup times. A cloud-based data management system collects monitored machine tool and process data, and is at the same time able to return performance predictions. In the course of two use cases from aerospace and automotive sector, predictive maintenance and a correlation between process parameters and quality measurement is demonstrated [Armendia 2019b].

Möhring et al. introduce the idea of self-optimizing machining systems (SOMS), which combine several elements of industry 4.0 for the adaptive machining of workpieces [Möhring 2020]. Elements of SOMS are process planning, monitoring, adaptive control, simulation and $\mathrm{Al}$ solutions. Focus is on the integration of elements with each other for the application in different machining cases.

Hänel et al. demonstrate the acquisition of planning and manufacturing data along with an integration of a cutting force model for the establishment of a digital process twin [Hänel 2019]. An information model based on UML class diagram along with HDF data storage is proposed.

Overall, only a limited amount of research work regarding digital twin frameworks can be found in the machining domain. Existing research provides promising approaches in partial areas of machining. However, a holistic concept and an exemplary implementation of a digital twin framework for the machining domain, which applies a wide range of current 14.0 technologies, is missing.

\section{DIGITAL TWIN FRAMEWORK FOR THE MACHINING DOMAIN}

The proposed digital twin framework for the machining domain represents a domain-specific implementation of a big data lambda architecture [Marz 2015], combined with the draft ISO 23247 (automation systems and integration digital twin framework for manufacturing) [ISO 23247]

The lambda architecture, initially proposed by Nathan Marz, comprises two types of data flows and four main elements (see Figure 1):

- Serving layer: Storage and provision of processed data in case of a query from a service or a user

- Batch layer: Batch-wise, non-real time processing of large resting data sets (also referred to as "cold path")

- Stream layer: Low-latency, real-time or close to realtime processing of small, fast moving data sets (also referred to as "hot path")

- Data source: Provision of data from databases (batch layer) or data streams (stream layer)

\begin{tabular}{|c|c|}
\hline \multicolumn{2}{|l|}{ Lambda architecture } \\
\hline \multicolumn{2}{|c|}{ Serving layer } \\
\hline 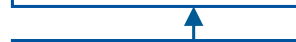 & 4 \\
\hline $\begin{array}{l}\text { Batch layer } \\
\text { (cold path) }\end{array}$ & $\begin{array}{l}\text { Stream layer } \\
\text { (hot path) }\end{array}$ \\
\hline \multicolumn{2}{|c|}{ Data source } \\
\hline
\end{tabular}

Figure 1: Lambda architecture

The reason for choosing a lambda architecture is its batch layer, which is eminent for processing the large data sets in machining (e.g. millions of CNC data points throughout a single machining operation) with the complex technology models of the machining domain (e.g. dexel-based numerical engagement simulation). At the same time, the speed layer is able to process small and sometimes fast moving data sets for monitoring and control purposes, such 
as temperature, acoustic emission or coolant sensor data. During the implementation of a domain-specific digital twin framework for machining, the big data technology stack of the lambda architecture has to be integrated with the domain-specific resources, applications and services of the machining domain, such as CAx and many other.

The general digital twin framework for manufacturing of ISO 23247 divides the manufacturing domain into four main areas, namely user domain, core domain, data collection and device control domain, as well as observable manufacturing domain (see Figure 2):

- User Domain: Human, device, application, system that uses applications and services from core domain

- Core Domain: Operation and management of digital twin (provisioning, monitoring, optimization etc.)

- Data collection and device control domain: Monitoring and collection of data from sensory devices

- Observable manufacturing domain: Physical manufacturing resources such as personnel, equipment, material, etc.

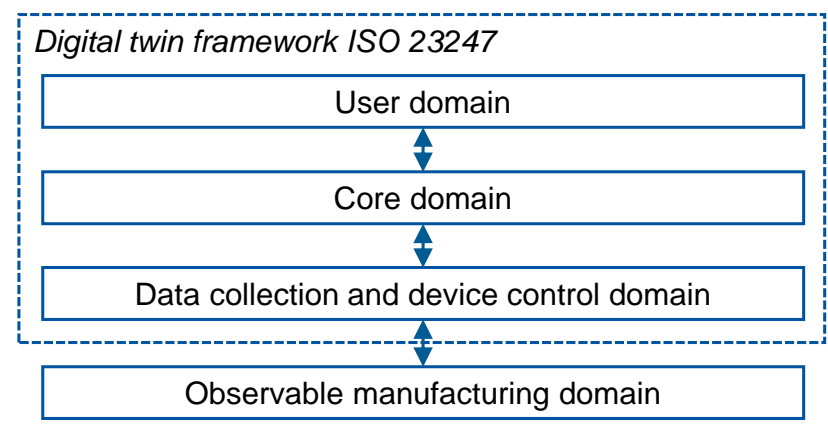

Figure 2: Digital twin framework ISO 23247

Figure 3 illustrates the combination of the lambda architecture with the domain-based reference model of ISO 23247, as well as mapping of the specific resources, applications and services of the machining domain onto the framework. The following subsections explain the domainspecific implementation of the framework.

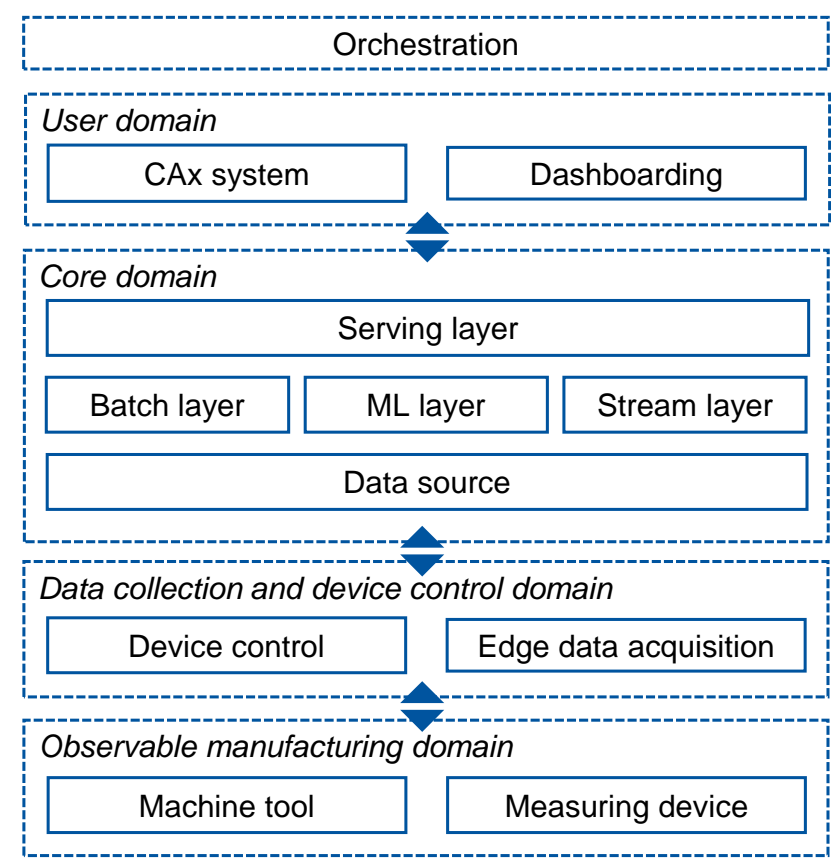

Figure 3 : Proposed digital twin framework for the machining domain

\subsection{Orchestration}

The implementation of the proposed digital twin framework operates on local, edge or cloud devices, across a variety of different applications and data types. Therefore, an orchestration layer is required, which manages the underlying framework elements (especially cloud elements; see Figure 4). Furthermore, it specifies the required semantic data model, which is required to merge all crosssystem data into a single digital twin.

\section{Cloud infrastructure and loT management}

The underlying cloud infrastructure is an "International Data Spaces (IDS) ready" on-premise cloud solution of the Fraunhofer-Gesellschaft (Fraunhofer Edge Cloud FEC). The FEC infrastructure at the Aachen, Germany institute node is an ONCITE base brick with 208 cores and 1.5 TB RAM, along with an ONCITE storage brick of $27 \times 10$ TB HHD raw storage. Further cloud nodes currently exist at Ilmenau and Berlin, Germany. The software stack comprises OpenStack as cloud platform, Kubernetes for cluster management and Docker for the containerization of applications. Furthermore, the on-premise cloud provides different zones, an internal, a shared, and a public zone, thereby allowing the joint development and operation of applications together with external partners.

Semantic data model

For a complete and data-consistent digital twinning across different applications and data types, we propose domain ontologies, and use these with persistent identifiers (PIDs) [Schiller 2021]. The architecture is based on the Resource Description Framework (RDF) that offers both ingestion and utilization of valuable information along the manufacturing steps within the product lifecycle. We might integrate aspects of the upcoming topic of asset administration shells (AAS) to the semantic data model in the near future.
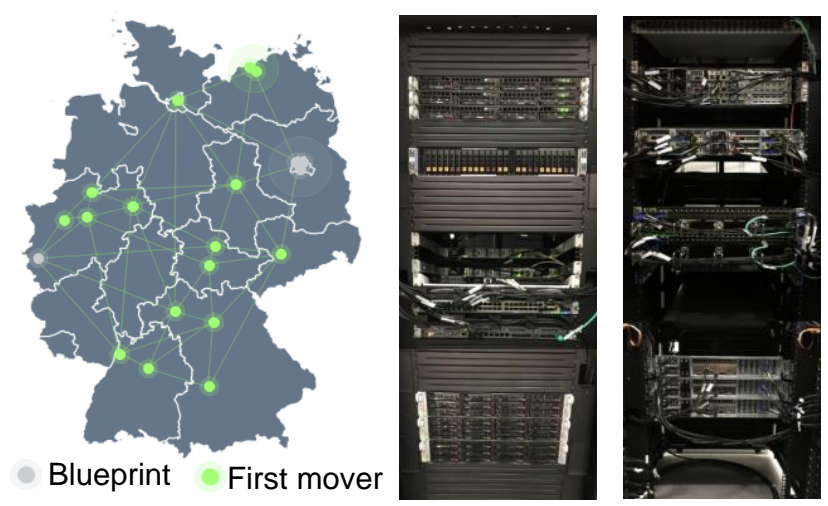

\section{Orchestration}

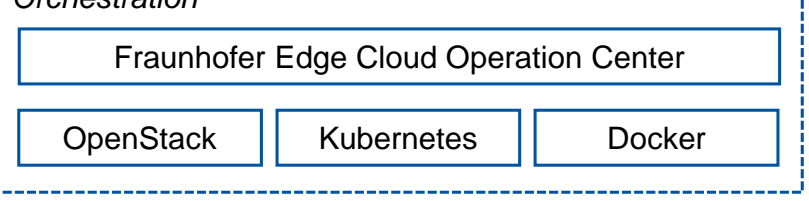

Figure 4: Fraunhofer on-premise cloud infrastructure

\subsection{User domain}

Regarding the user domain, we propose two locations for user interaction with the digital twin. Due to its established role within the product lifecycle and its technical maturity, we recommend the CAx system as the main environment for direct user interaction with the digital twin. Furthermore, we recommend lightweight interfaces in the form of dashboards for interacting with the digital twin within web applications or on mobile devices. 


\section{CAx system}

Today's CAx system is a local desktop application with low computing power as well as a lack of integrated and coupled technology models for process simulation. In addition to outstanding development work at the core of the systems, the shortcomings are also based on missing networking capabilities of the systems with modern edge or cloud ecosystems. Because of the technical inadequacies, insufficient enrichment of the digital twin with simulation data takes place during the process planning phase. Furthermore, no manufacturing data is returned to the CAx system after production.

In order to enhance the completeness of the digital twin in the process planning phase, we tightly integrate our proprietary computer-aided engineering (CAE) kernel for process simulation. Figure 5 (bottom) shows the functionalities of the CAE kernel, respective technology models. A commercial CAD system with a wide application architecture and open SDK functions is used as the basic environment. We extend the CAD environment with a proprietary CAM plug-in and our proprietary CAE plug-in for process simulation in the form of dynamic link libraries (DLL). The integration comprises software interfaces between the CAD/CAM/CAE kernels as well as adaptions of the CAx user interface. Although one might assign the $\mathrm{CAD} / \mathrm{CAM} / \mathrm{CAE}$ kernels of the local CAx system to the core domain, we assign it to the user domain in this case.

Furthermore, we connect the CAx system to our FEC cloud infrastructure through a web server, enabling an extension to the core domain. A detailed explanation of the extension is given in section 3.3.

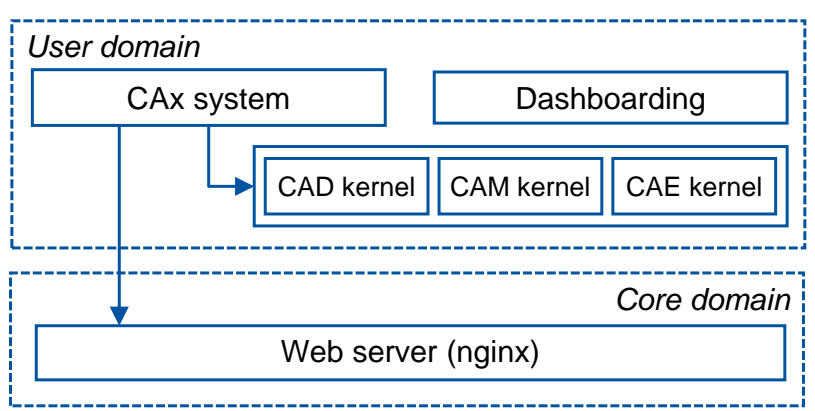

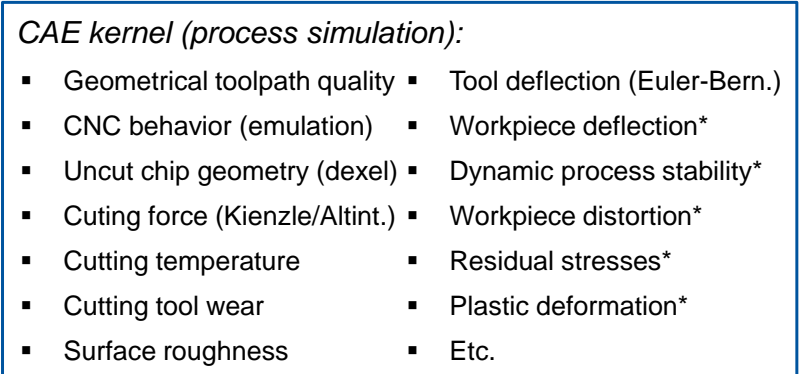

*Incl. Finite Element Method (FEM) interface

Figure 5: CAE kernel integration and and cloud interface of the CAx system

\section{Dashboarding}

For some applications, the targeted visualization and assessment of specific quality, time, cost or ecology information might be of relevance. Furthermore, access to a full CAx system is difficult to realize at every location and for every person within manufacturing. Interaction with the digital twin via dashboards and mobile devices represents a suitable alternative. Such dashboards can be built efficiently from open source platforms (React, Angular etc.)

\subsection{Core domain}

The functional elements of the core domain can be executed either local, on the edge, or on cloud level. In our understanding, most of the functional elements of the core domain should be executed in the cloud. The following section explains the data provision to the user domain over a serving layer, the domain-specific data processing via batch, ML and stream layer, as well as the data sources of the core domain (see Figure 6).

\begin{tabular}{|c|c|c|}
\hline \multicolumn{3}{|l|}{ Core domain } \\
\hline \multicolumn{3}{|c|}{ Serving layer } \\
\hline \multicolumn{3}{|c|}{ Analytics data store (MongoDB) } \\
\hline Batch layer & ML* layer & Stream layer \\
\hline $\begin{array}{l}\text { Technology } \\
\text { microservices }\end{array}$ & ML model & $\begin{array}{l}\text { Stream analytics } \\
\text { pipelines }\end{array}$ \\
\hline $\begin{array}{l}\text { (Based on CAD, } \\
\text { CAM, CAE etc.) }\end{array}$ & deployment & $\begin{array}{c}\text { (Apache Spark, } \\
\text { Apama etc.) }\end{array}$ \\
\hline \multicolumn{3}{|c|}{ Data source } \\
\hline Database (MDB) & Broker (MQTT) & Broker (Kafka) \\
\hline
\end{tabular}

\section{Figure 6: Core domain elements of the digital framework}

\section{Serving layer}

The main component of the serving layer is an analytics data store in the form of a NoSQL database (MongoDB). It holds the computed batch and stream views of the core domain. Different files (TDMS, JSON, Parquet, STEP etc.) and their relation based on the semantic data model represent the digital twin. Different entities or users in the user domain, e.g. the CAx system, can query the digital twin information from the serving layer.

Batch layer

The batch layer contains a large number of technology microservices. These microservices are technologyspecific data processing applications in the form of Docker images, mainly build from the CAD/CAM/CAE kernel functionalities of the machining domain. The technology microservices compute process or workpiece condition data, thereby providing digital twin information in the form of batch views to the serving layer.

As an example, Figure 7 shows the extension of the CAx system into the core domain. In order to increase the data storage, processing and analysis capabilities of the CAx system, it is connected to a web server (nginx) in the FEC cloud infrastructure. The web server transmits CAx input information in the form of different data types (JSON, STEP, STL etc.) to a web framework (Django / Node.js). The web framework is connected to a NoSQL database (MongoDB) along with a simulation scheduler, which again operates a number of workers. The workers can execute multiple instances of the CAD/CAM/CAE kernels in the form of Docker images on virtual machines, thereby significantly increasing the computing power of the CAx system through parallelization.

The integration of the extended process simulation capabilities along with the increased computation power based on the cloud interface allows for complex process simulations, parallelized process optimizations and many 
more. Both factors significantly enhance the completeness of the digital twin in the process planning phase. Other batch applications, e.g. the edge data acquisition and subsequent data processing, explained in the following section 3.4, operate on principally the same architecture.

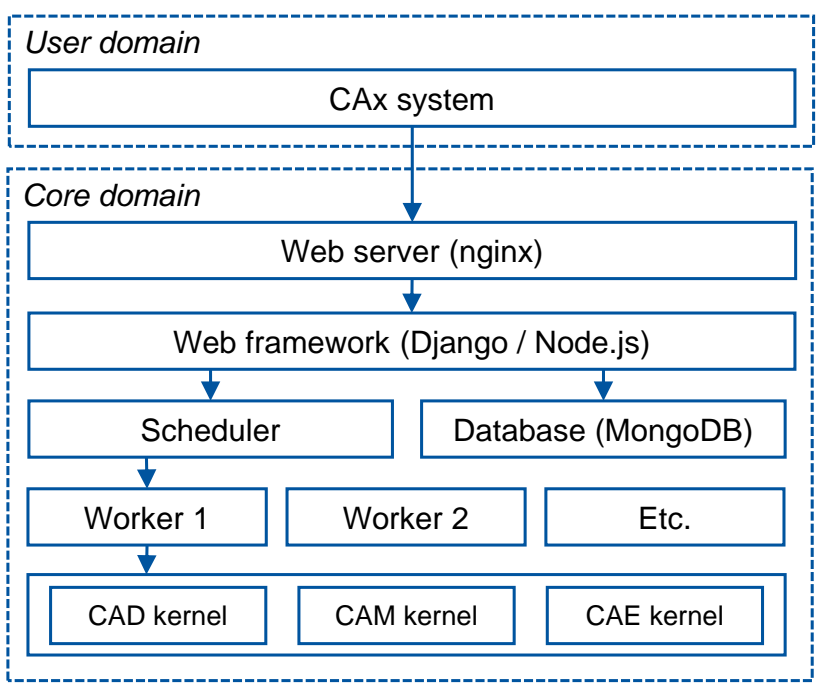

Figure 7 : Exemplary extension into the core domain (here of the CAx system)

\section{Machine learning layer}

We extend the lambda architecture with a machine learning layer. The ML layer takes up data from the batch and stream layer to train ML models and deploy them to the batch and stream layer for data processing. An example is our digital image processing with deep learning for automated cutting tool wear detection [Bergs 2020b]. In this case the ML model is trained in the ML layer with acquired tool wear information from a machine integrated camera in the observable manufacturing domain. The trained model is then integrated into the batch and stream layer for tool wear quantification.

\section{Stream layer}

The stream layer consists of streaming analytics pipelines, which can be established from platforms such as Apache Spark or Apama. Exemplary streaming analytics applications are for example the monitoring of the spindle power or the coolant concentration over a sliding time window. The streaming data is used to update the batch views in the serving layer or is directly streamed to the user domain for evaluation.

\section{Data source}

In general a persistent NoSQL database, along with a Message Queuing Telemetry Transport (MQTT) and Apache Kafka broker, provide acquired raw data, structured per machining operation, to the batch, ML and stream layer for data processing. The acquired raw data resembles the digital shadow of the manufactured workpiece and is converted to the digital twin inside the batch, ML and stream layer through data processing.

\subsection{Data collection and device control domain}

The data collection and device control domain is supposed to monitor, control and collect data from the elements of the observable manufacturing domain. Concerning the implemented framework, this primarily comprises an edgebased data acquisition solution (see also Figure 8).

Edge data acquisition:

Today's machine tools exchange data with other systems in the manufacturing environment only to a limited extent.
The high frequency and time synchronous acquisition of machine and sensor data on field and control level via suitable edge devices is not industrial state of the art. Accordingly, the digital twin can only insufficiently be supplemented with manufacturing data (acquired meta and raw data, model-based determined process and product condition data) during the manufacturing phase.

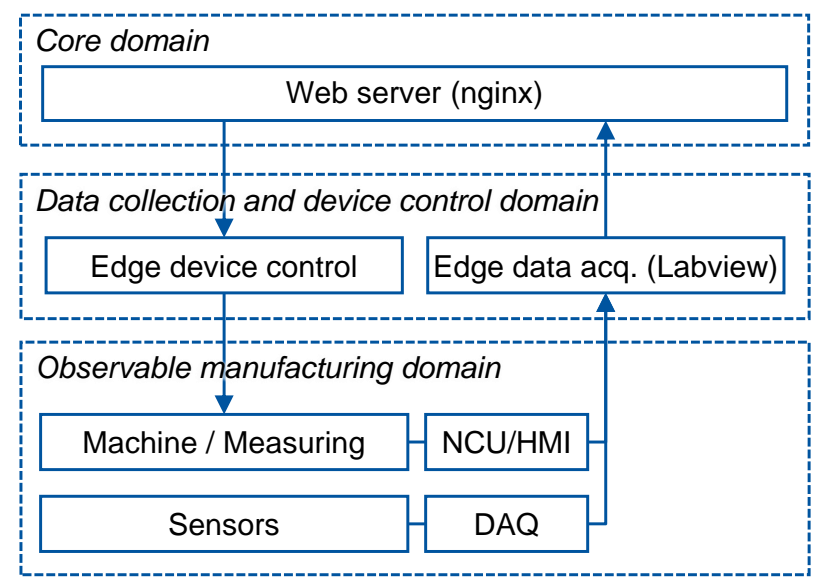

\begin{tabular}{|c|c|c|}
\hline \multicolumn{3}{|c|}{ Edge data acquisition: } \\
\hline - Axis speed & - Tool length $Y$ & - Offset G54 \\
\hline - Feed speed & - Tool length Z & - Axis names \\
\hline - Operation type & - Tool radius & - Program name \\
\hline - Spindle speed & - Cutting edge & - R parameter \\
\hline - Position in WCS & - Cutting edge & - Position in MCS \\
\hline - Tool name & moditıed & - UGUDs \\
\hline - Tool length X & - Axis loads & - Etc. \\
\hline
\end{tabular}

Figure 8 : Data collection and device control domain infrastructure

To overcome the described deficit in machining, we introduce an edge data acquisition. The solution is a proprietary LabVIEW application for data acquisition on HMI task level, integrating commercially available access libraries depending on the control type (Siemens 840D sl / Fanuc Professional 6). It is able to maintain a stable data acquisition frequency of $>250 \mathrm{~Hz}$ across a maximum of 100 channels. It integrates and synchronizes additional sensor data from multiple devices, such as force, acoustic emission and temperature sensors or cameras. Integration of the additional sensors takes place in the machine or the clamping devices, tool holders etc. The acquired data is stored in different file formats (TDMS, JSON, Parquet etc.).

\subsection{Observable manufacturing domain}

The observable manufacturing domain of machining mainly comprises the machine tool, which executes the machining operation. Additionally, the measuring devices from quality assurance such as coordinate measuring machines (CMM) might be included.

Machine tool:

The previously introduced edge data acquisition has been integrated into four machine tools (Mikron HPM800U, DMG MORI DMU65FD, Hermle C42MT, Makino D500). The required edge device is an industrial personal computer (SIMATIC IPC677E), installed in the control cabinet of the machine tool (see Figure 9). Connection is realized on human-machine interface (HMI) task level via TCP/IP ethernet connection to the numerical control unit (NCU). Figure 9 shows the installed I-PC, along with a control cabinet for temperature and acoustic emission sensor data 
acquisition (DAQ chassis), inside one of the connected machine tools.

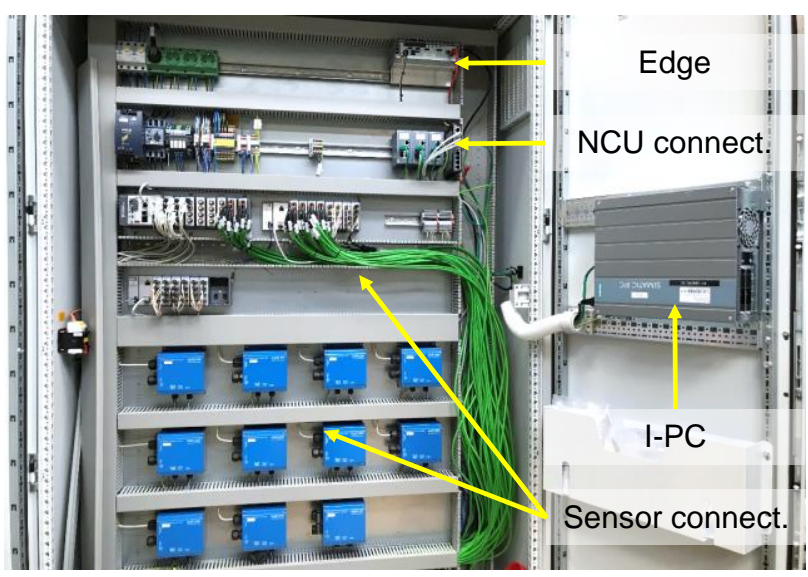

Figure 9: Edge data acquisition hardware

\section{FRAMEWORK APPLICATION}

In order to demonstrate the application of the implemented digital twin framework, this chapter introduces two use cases. The first use case is a process design task, the second use case is a quality prediction task, both in milling of a blade integrated disk (BLISK) demonstrator. Figure 10 shows the most relevant demonstrator characteristics.

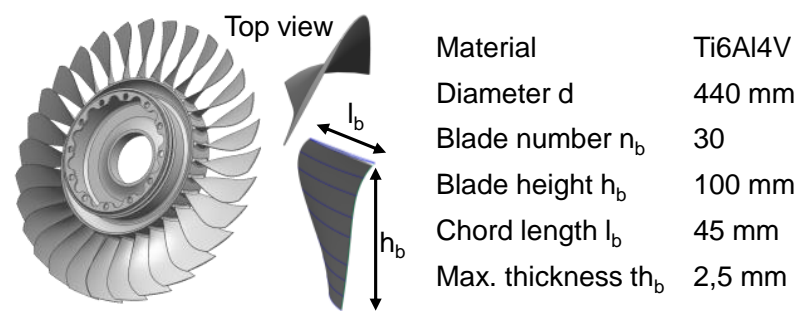

Figure 10 : Blisk demonstrator

A seven-block strategy was selected for blade machining to limit the free overhang and deflection of the blades during milling. Three different solid carbide tools were used for the 5-axis milling of the blades: (1) roughing tool: $\varnothing 16 \mathrm{~mm}$ bull nose; (2) semi-finishing tool: $\varnothing 16 \mathrm{~mm}$ ball end; (3) finishing tool: $\varnothing 12 \mathrm{~mm}$ ball end, cantilever length $\mathrm{L}_{w}=105 \mathrm{~mm}$.

\subsection{Use case I: Process design}

In a possible process design task a user would like to determine the correlation between the step-over $a_{e, S F}$ in semi-finishing and the tool deflection $\Delta_{t r}$ in finishing. Beyond CAD/CAM functionalities, the described process design task requires integrated process simulation capabilities along with sufficient computation power. However, today's CAM system provides no support functions of that type, especially not based on a digital twin representation.

In order to fulfil the above mentioned process design task, we created input information in the local CAx system, respective the user-domain:

- Seven semi-finishing and finishing operations, one for each block of the blade (+ corresponding binary files for reproduction on cloud level)

- CAM parameter variation values $\left(\mathrm{ap}_{\mathrm{p}, \mathrm{SFi}}\right)$

- JSON array of characteristic simulation parameters

- Macroscopic tool geometry information

- Elastic modulus of solid carbide: $E_{t}=600 \mathrm{GPa}$

- Force coefficients:
$\mathrm{K}_{\mathrm{tc}}: 2746 \mathrm{~N} / \mathrm{mm}^{2}, \quad \mathrm{~K}_{\mathrm{rc}}: 981 \mathrm{~N} / \mathrm{mm}^{2}, \mathrm{~K}_{\mathrm{ac}}: 14 \mathrm{~N} / \mathrm{mm}^{2}$, $K_{\text {te: }}: 14 \mathrm{~N} / \mathrm{mm}, K_{\text {re: }}: 55 \mathrm{~N} / \mathrm{mm}$, Kae: $55 \mathrm{~N} / \mathrm{mm}$

- Raw and finish part geometry

The CAx system sent a simulation request to cloud level, respective the core-domain, via the web server and uploaded all required input information to the database. A request handler checked whether a similar simulation request was made in the past and whether corresponding simulation data was already available. It communicated a data generation command to the simulation scheduler. The simulation scheduler sorted the simulation request by computational effort and managed the overall optimization workflow. In this case, three simulation chains were generated and divided into 42 subtasks for computational parallelization, resulting from the combination of 3 variations of semi-finishing operations in 7 different blocks each, followed by a subsequent finishing operation. The system started a corresponding number of simulation workers to execute the CAD/CAM/CAE kernels on virtual machines consisting of five modularly built and interconnected Docker Images:

1. CAM Toolpath calculation

2. Macroscopic engagement simulation (multi-dexel)

3. Microscopic engagement simulation (analytical)

4. Dual-mechanistic cutting force simulation (Altintas)

5. Tool deflection simulation (Euler-Bernoulli)

Subsequently, the simulation workers saved all process and part condition data in the analytical data store of the core domain. Based on the parallelized system the entire simulation task could be performed below one hour, instead of days, and could thereby be integrated into the regular CAx workflow. Figure 11 shows the digital twin information, which was returned from the analytical data store to the local CAx system.
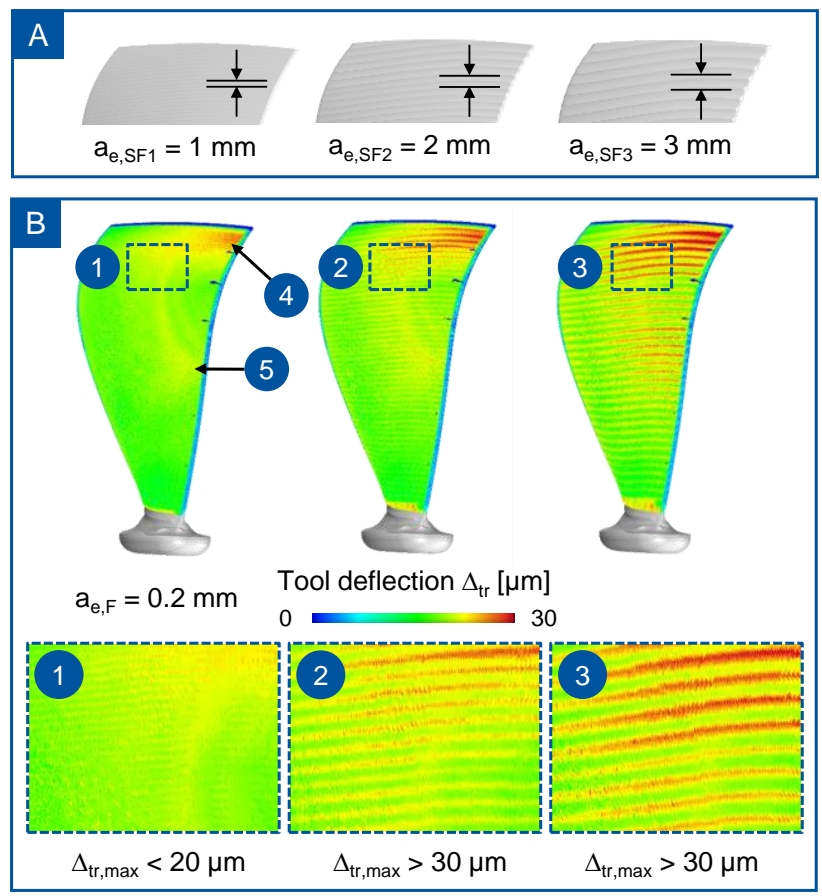

Figure 11 : Semi-finishing IPWs after variation of step-over $a_{e, S F}(A)$; Resulting tool deflection $\Delta_{t r}$ in finish milling $(B)$

Figure $11(\mathrm{~A})$ shows the resulting in-process workpieces (IPWs) which result from semi-finishing with the three different step-over values ae,SFi. Figure 11 (B) shows the 

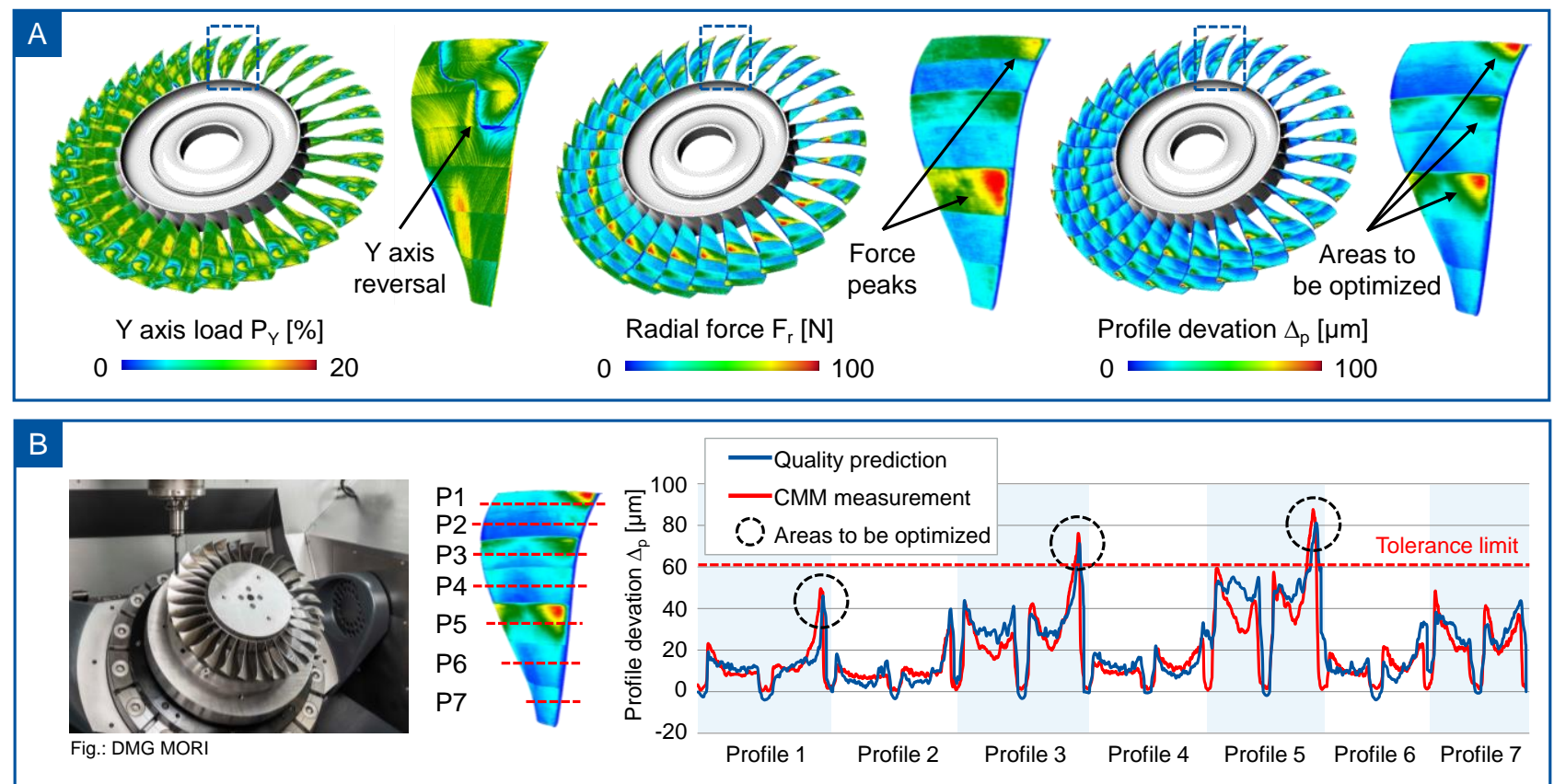

Figure 12: Digital twin carrying machine, process and part condition data (A); Comparison of predicted and measured profile deviations of the manufactured blisk component $(B)$

corresponding tool deflection after finish milling with a step-over of $\mathrm{a}_{\mathrm{e}, \mathrm{F}}=0.2 \mathrm{~mm}$. Within the areas 1-3 it can be clearly seen, that a semi-finishing with $a_{e}, \mathrm{SF}>1 \mathrm{~mm}$ results in excessive tool deflections $\Delta_{t r}>20 \mu \mathrm{m}$ during finish milling. The results are an indication for a user to not exceed $a_{p, S F}=1 \mathrm{~mm}$ as parameter for the semi-finishing process. Furthermore, for all three cases noticeable force peaks occur at the blade tip close to the leading edge (4), as well as at half of the blade height close to the leading edge (5). The user might later identify these force peaks also in the real manufacturing process.

\subsection{Use case II: Data-driven quality prediction}

The second use case demonstrates the quality prediction from machine and sensor data directly in manufacturing. The prediction of the workpiece quality during or right after completion of the manufacturing process enables the reduction of inspection efforts in the later quality assurance, and an in-depth process investigation and optimization.

The previously introduced blisk demonstrator was manufactured on a DMG MORI DMU 65FD machine tool. The machine was equipped with a Siemens Sinumerik 840D sl v4.8 control and an integrated Tool Control Center (TCC) for the measurement of bending moments and axial forces acting on the spindle. The production was carried out according to the milling strategy described at the beginning of chapter 4. After manufacturing the blisk was measured on a Zeiss Prismo CMM with a rotary table. The profile deviations were measured at seven cross-sections at half of the height of each block for each blade (see Figure 12).

Extensive process data acquisition took place as part of the investigation. Machine-internal signals, such as the position data of the axes, as well as the load signals of all drives were recorded by the edge data acquisition at the machine tool at a frequency of approximately $250 \mathrm{~Hz}$. In addition, the software carried out the acquisition of critical metadata such as workpiece identification data, NC-program structure and tool parameters. The signals of the machine integrated TCC were acquired synchronously from the edge data acquisition via an MQTT and UDP interface at a frequency of $2500 \mathrm{~Hz}$. The data acquisition software generated files in parquet format (batches), each assigned to an operation, and sent them to the core domain scheduling services via MQTT protocol. The results of the subsequent CMM inspection process were exported as CSV files and imported into the digital twin framework via a microservice running on a Siemens SIMATIV 127e IPC. The CSV file contained the points of the measured profiles and their deviation from the nominal.

Inside the core domain, all data dispatched by the edge data acquisition was processed in the batch layer. Each captured batch was prepared and scheduled for analysis by a scheduling service. The data was then resampled and processed by a technology microservice. The service performs geometrical operations, such as kinematic transformations, and reproduces the physical manufacturing process and its technological effects, such as tool and workpiece deflection or tool wear etc.. It thereby translates the acquired machine and sensor data into process and workpiece condition data. The generated results were stored in the analytical data store in the form of parquet files.

Figure 12 shows the digital twin information, which was returned to the user domain. Part $A$ of the figure shows the $Y$ axis load of all finishing operations. As an example, axis reversals can be identified from the image, which resulted in slight notches on the component. Part B of the figure shows the cutting force calculated from the bending moment recorded by the TCC during finishing. The cutting force is in the range of 0-90 N. The force signal shows the same force peaks at the blade tip and half of the blade height, which were already identified in the process design use case. Part $C$ of the figure shows the calculated profile deviations of the blisk component. The profile deviations are in the range of $0-100 \mu \mathrm{m}$ and indicate the need for further process optimizations in three positions close to the leading edge of the blade.

In order to evaluate the resulting quality prognosis, CMM measurements were carried out and used for validation. The measured points contained in the CMM result data were associated with the results of the quality prediction by a microservice in the batch layer. Figure 12 shows the good agreement of the quality prediction with the CMM measurement across the seven profiles for the first blade. 
An average deviation of $-1 \mu \mathrm{m}$ to $+5 \mu \mathrm{m}$ was achieved between measurement and prognosis across the seven measured profiles. Depending on the crosssection, the standard deviation was between 3-4 $\mu \mathrm{m}$. Remaining deviations are due to possible errors in the measurement, e.g. probe radius compensation or technological effects not taken into account in the analysis.

\section{OUTLOOK}

Continuous further development of the presented digital twin framework and its implementation will take place. Major focus will be on the area of orchestration, such as the semantic data model and the integration of asset administration shells (AAS). Furthermore, focus will be on the implementation of advanced streaming analytics pipelines and machine learning applications inside the stream and ML layer of the lambda architecture.

\section{SUMMARY}

The presented paper introduces a digital twin framework for the machining domain, along with an exemplary implementation of the major functional elements. The framework is a combination of a big data lambda architecture and the digital twin framework of ISO 23247. The implementation comprises specific solutions in the area of process simulation, cloud infrastructure, batch and stream data processing, as well as machine data acquisition. We have presented first batch processing applications in the area of process design and predictive quality. The use cases clearly illustrate, that the presented framework implementation is a key enabler on the way to a complete and data-consistent digital twin in machining.

\section{ACKNOWLEDGMENTS}

The process design use case was funded by the federal ministry of education and research in the research project "CAM2030 « (BMBF 02J19B080). The predicitive quality use case was funded by the federal ministry for economic affairs and energy in the EXIST transfer of research "Quantify« (BMWi 03EFQNW272). Furthermore, we acknowledge the support of DECKEL MAHO Pfronten, German Edge Cloud, ModuleWorks and pro-micron.

\section{REFERENCES}

[Abele 2016] Abele, E. (ed.) WGP-Standpunkt Industrie 4.0. Frankfurt am Main, 2016.

[acatech 2011] acatech (ed.) Cyber-Physical Systems. Springer, Berlin, Heidelberg, 2011.

[Altintas 2014] Altintas, Y., et al. Virtual process systems for part machining operations. CIRP Annals, 2014, Vol. 63, No. 2, pp. 585-605.

[Armendia 2019a] Armendia, M., et al. Evaluation of Machine Tool Digital Twin for machining operations in industrial environment. Procedia CIRP, Vol. 82, 2019, pp. 231-236. ISSN 2212-8271

[Armendia 2019b] Armendia, M., et al. (ed.) Twin-Control. Springer International Publishing, 2019. ISBN 978-3-03002203-7

[Bergs 2020a] Bergs, T., et al. (ed.) Mit dem Digitalen Zwilling Prozessgrenzen überwinden. Statusberichte aus der Produktionstechnik 2020.

[Bergs 2020b] Bergs, T., et al. Digital image processing with deep learning for automated cutting tool wear detection. Procedia Manufacturing, Vol. 48, 2020, pp. 947-958. ISSN 2351-9789
[Bergs 2021] Bergs, T., et al. The Concept of Digital Twin and Digital Shadow in Manufacturing. Procedia CIRP, 2021 [Boje 2020] Boje, C. et al., Towards a semantic Construction Digital Twin: Directions for future research. Automation in Construction, Vol. 114, 2020, 103179. ISSN 0926-5805

[Brecher 2011] Brecher, C., Wolfram, L., A CAM-integrated Virtual Manufacturing System for Complex Milling Processes. Proceedings of the 44th CIRP International Conference on Manuf. Systems, 2011, Madison, WI, USA.

[Caesar 2020] Caesar, B., et al., Information Model of a Digital Process Twin for Machining Processes. IEEE International Conference on Emerging Technologies and Factory, 2020, Vienna, Austria.

[Croatti 2020] Croatti, A., et al., On the Integration of Agents and Digital Twins in Healthcare. J Med Syst 44, 161, 2020. [Geissbauer 2017] Geissbauer, R., et al., Digital Factories 2020: Shaping the future of manufacturing. PricewaterhouseCoopers GmbH Wirtschaftsprüfungsgesellschaft (PwC), 2017.

[Glaessgen 2012] Glaessgen, E., Stargel, D., The digital twin paradigm for future NASA and U.S. air force vehicles. Structural Dynamics and Materials Conference, 22267.

[Grieves 2015] Grieves, M., Digital Twin: Manufacturing Excellence through Virtual Factory Replication. Digital Twin White Paper, 2015.

[Haenel 2019] Haenel, A., et al. Development of a method to determine cutting forces based on planning and process data as contribution for the creation of digital process twins. MM Science Journal, 2019. ISSN 1803-1269

[Haenel 2020] Haenel, A., et al. The development of a digital twin for machining processes for the application in aerospace industry. Procedia CIRP, Vol. 93, 2020, pp. 1399-1404.

[ISO 23247] ISO/DIS 23247 Automation systems and integration - Digital Twin framework for manufacturing. Publication date: 2020-07 (draft).

[Kritzinger 2018] Kritzinger, W., et al. Digital Twin in manufacturing: A categorical literature review and classification. IFAC-PapersOnLine, Vol. 51, Issue 11, 2018, pp. 1016-1022. ISSN 2405-8963

[Marz 2015] Marz, N., Warren, J., Big Data. Manning, New York, 2015. ISBN 9781617290343

[Moehring 2020] Moehring, H.-C., et al., Self-optimizing machining systems. CIRP Annals, Vol. 69, Issue 2, 2020, pp. 740-763. ISSN 0007-8506

[Monostori 2014] Monostori, L., Cyber-physical Production Systems: Roots, Expectations and R\&D Challenges. Procedia CIRP, Vol. 17, 2014, pp. 9-13. ISSN 2212-8271

[Negri 2017] Negri, E., et al., A Review of the Roles of Digital Twin in CPS-based Production Systems. Procedia Manufacturing, Vol. 11, 2017, pp. 939-948. ISSN 23519789

[Schiller 2021] Schiller, S., et al., Towards Ontology-based Lifecycle Management in Blisk Manufacturing. 15th CIRP Conference on Intelligent Computation in Manufacturing Engineering, 2021 (abstract accepted).

[Teige 2021] Teige, C., Intelligente Vernetzung für die Fraesbearbeitung. wt Werkstatttechnik online, 2021.

[Wiederkehr 2016] Wiederkehr, P., Siebrecht, T. Virtual Machining: Capabilities and Challenges of Process Simulations in the Aerospace Industry. Procedia Manufacturing, Vol. 6, 2016, pp. 80-87. ISSN 2351-9789 